Aline Bruehmueller Ale Fernandes ${ }^{1}$

Igor Lucas Pinheiro de Sousa ${ }^{1}$

Jordana Gasparelo Santi ${ }^{1}$

Maristela da Silva Andreoni ${ }^{1}$

Paulo Luiz Batista Nogueira ${ }^{1}$

\section{Incidence of death due \\ to violent causes among underage individuals in Cuiabá City, Brazil, from 2015 to 2016}

\title{
Ocorrência de óbitos de causa violenta em menores de 18 anos, na cidade de Cuiabá, entre os anos de 2015 e 2016
}

ABSTRAT | Introduction: Death profile in children younger than

18 years in Brazil has considerably changed after the country's urbanization, since population increase was followed by intense criminality and individuals got more vulnerable to violence. Objective: The main objective of the current study is to analyze the frequency and features of violent deaths among children younger than 18 years, with emphasis on prevalent causes of death in general and in specific groups.

Methods: Cross-sectional descriptive retrospective epidemiological study was carried out at the Medical Examiner's Office (IML - Instituto Médico Legal) in Cuiabá City, Mato Grosso State, in 2015 and 2016. Results: Results have shown that external causes, mainly traffic accidents and gunshot wounds, accounted for most death cases. Male individuals in the age group 13-18 years recorded the highest death rates. Conclusion: Increased number of deaths due to violent causes can be explained by the fact that young individuals are increasingly

exposed to crime events, such as physical assaults, gunshots and accidents, early in life. Non-white male individuals in the age group 13-to-18 years are mostly vulnerable to these causes of death. In addition to violence, children and adolescents have been victims of accidents, mainly of traffic accidents, which accounted for $21.5 \%$ of causes of death.

Keywords | Mortality; Children; Adolescents; External causes; Forensic Medicine.
RESUMO| Introdução: O perfil de óbitos em menores de 18 anos no Brasil mudou de forma considerável após a urbanização do País, quando com o aumento populacional a criminalidade também começou a se intensificar, e os indivíduos passaram a ser mais vulneráveis à violência. Objetivo: $\mathrm{O}$ principal objetivo deste estudo é analisar a frequência e as características de mortes violentas em menores de 18 anos, ressaltando as causas predominantes de forma geral e específicas para grupos de indivíduos. Métodos: Foi realizado um estudo epidemiológico descritivo do tipo transversal, retrospectivo, no Instituto Médico Legal (IML) da cidade de Cuiabá, no estado do Mato Grosso, nos anos de 2015 e 2016. Resultados: Os resultados demonstraram que as causas de morte externa têm sido responsáveis pela maioria dos óbitos, especificamente os acidentes de trânsito e a perfuração por arma de fogo. O maior percentual foi de indivíduos entre 13 e 18 anos e do sexo masculino. Conclusão: $\mathrm{O}$ aumento de óbitos por causas violentas tem sido explicado pelo fato de os jovens estarem em contato com a criminalidade cada vez mais precocemente, sendo expostos a agressões, perfuração por arma de fogo e acidentes com frequência. Os não brancos, do sexo masculino, que estão na faixa etária de 13 a 18 anos, são os mais vulneráveis a essas causas de morte. Além da violência, as crianças e adolescentes vêm sendo vítimas dos acidentes, especificamente de trânsito, responsáveis por 21,5\% das causas de óbito.

Palavras-chave | Mortalidade; Crianças; Adolescentes; Causas externas; Medicina Legal.

${ }^{1}$ Centro Universitário de Várzea Grande. Várzea Grande/MT, Brasil. 


\section{INTRODUÇÃO|}

O perfil de óbitos em menores de 18 anos no Brasil mudou de forma considerável após a urbanização do País, quando com o aumento populacional a criminalidade também começou a se intensificar, e os indivíduos passaram a ser mais vulneráveis à violência ${ }^{1}$. As doenças do aparelho circulatório, as neoplasias e as causas externas se tornaram, nessa ordem, responsáveis pela maioria das mortes nessa faixa etária da população brasileira ${ }^{2}$. Desde a década de 1980 , as mortes por violência e acidentes vêm crescendo significativamente e se tornaram uma preocupação para a saúde pública ${ }^{3}$.

Como consequência, em 1990, começaram a ser criadas medidas de proteção para crianças e adolescentes. Assim, em 13 de julho de 1990, obedecendo ao art. 227 da Constituição Federal, foi aprovada a Lei Federal n. 8.069, o Estatuto da Criança e do Adolescente (ECA ${ }^{4}$. O ECA contém capítulos que asseguram, dentre outros, o direito à vida, saúde, liberdade, dignidade e até mesmo medidas de proteção para indivíduos entre 12 e 18 anos. Esperavase que, com essa legislação vigente, as mortes por causas violentas diminuíssem entre os menores de 18 anos.

De acordo com o Sistema de Informação sobre Mortalidade (SIM) do DATASUS², em 2016 foram mais 155 mil mortes por causas externas no Brasil, sendo que 21 mil indivíduos eram menores de 19 anos. Ainda nessa faixa etária e no mesmo ano, mais 11 mil indivíduos vieram a óbito por agressões, 900 por lesões autoprovocadas voluntariamente e aproximadamente $7 \mathrm{mil}$, por acidentes de transporte ${ }^{5}$. Observou-se também como fator de risco para a morte de causa violenta: ser do sexo masculino, estar na faixa etária de 10 a 18 anos, de baixa renda e não branco ${ }^{1,6}$.

Caracterizar os óbitos por causas externas se faz necessário não somente para refletir sobre a violência entre os menores de 18 anos, mas também para que se tomem medidas para assegurar os direitos dessa população, com mais ênfase no direito à vida e na prevenção de mortes por atos violentos e acidentes.

A realização desta investigação foi motivada por ser uma realidade para a saúde pública e devido à carência de estudos atuais sobre o tema. Dessa forma, o principal objetivo desse estudo é analisar a frequência e as características de mortes violentas em menores de 18 anos, ressaltando as causas predominantes de forma geral e específicas para grupos de indivíduos.

\section{MÉTODOS|}

Trata-se de um estudo epidemiológico descritivo do tipo transversal, retrospectivo. Os dados, referentes aos anos de 2015 e 2016, foram coletados em agosto de 2016, no Instituto Médico Legal (IML) da cidade de Cuiabá, no estado do Mato Grosso.

A revisão dos laudos médico-legais foi efetivada pelos próprios autores, totalizando 123 laudos, e abrangeu os óbitos por causas externas em menores de 18 anos necropsiados no mesmo IML, tendo como critérios de exclusão outras faixas etárias e óbitos fora do período avaliado.

Os dados foram submetidos a uma análise estatística descritiva e analítica, realizada pelo programa Epi Info versão 7 (Center for Diseases Control and Prevention) 2016 e transferidos para planilhas do Microsoft Excel para construção de gráficos e tabelas, contendo números absolutos e percentuais.

As variáveis analisadas foram a idade, sexo, cor da pele, causa da morte, circunstância, local e histórico do óbito. Ademais, buscou-se detalhar óbitos causados por traumatismo.

O estudo foi submetido ao Comitê de Ética da Universidade de Cuiabá e autorizado pelo parecer de número 2.110.470.

\section{RESULTADOS |}

No período estudado, foram analisados 123 laudos de necropsia em menores de 18 anos, em que 66,67\% eram do sexo masculino. Já em relação à faixa etária, o maior número de óbitos se deu entre 13 e 18 anos (60,98\%), seguindo de forma decrescente, de 1 a 3 anos (17,89\%), menores de 1 ano (9,76\%), de 7 a 12 anos (8,13\%) e com o menor percentual a faixa etária de 4 a 6 anos $(3,25 \%)$.

As vítimas fatais de mortes violentas foram, em sua maioria, de cor parda (65,04\%), seguida dos brancos e depois negros. Os óbitos de pardos e negros totalizaram $76,42 \%$, foi aproximadamente quatro vezes mais frequente que os de cor branca $(19,51 \%)$ (Tabela 1$)$. 
Tabela 1 - Características sócio-demográficas dos óbitos por causas externas em menores 18 anos, analisados no IML de Cuiabá, entre 2015 e 2016

\begin{tabular}{lll}
\hline Variáveis & N & $\%$ \\
\hline Sexo & 82 & \\
\hline Masculino & 41 & 66,67 \\
Feminino & & 33,33 \\
\hline Faixa etária & 75 & 60,98 \\
13 a 18 & 22 & 17,89 \\
1 a 3 & 12 & 9,76 \\
Menor 1 ano & 10 & 8,13 \\
7 a 12 & 4 & 3,25 \\
4 a 6 & & \\
\hline Cor da pele & 80 & 65,04 \\
Pardo & 24 & 19,51 \\
Branco & 14 & 11,38 \\
Negro & 5 & 4,07 \\
\hline Sem identificação & & \\
\hline
\end{tabular}

As principais causas de óbitos identificados no estudo foram por traumatismo crânioencefálico (31,71\%), seguidas por asfixia $(28,46 \%)$ e choque hipovolêmico $(15,45 \%)$. Segundo o histórico do óbito, 37 (30,08\%) vieram a óbito devido à lesão por arma de fogo, 27 (21,95\%) por acidente de trânsito e $16(13,01 \%)$ por afogamento, caracterizando os mais prevalentes. O local de maior ocorrência de óbitos foi em via pública $(66,67 \%)$ (Tabela 2$)$.

Ao relacionar a cor da pele e circunstância da morte, foi evidenciado que os brancos tiveram mortes de causa acidental em 41,67\% dos casos, morte natural em 20,83\%, homicídio em $16,67 \%$ e $8,93 \%$ suicidaram-se, sendo que $12,50 \%$ não foram identificados.

A circunstância acidental dos óbitos foi a mais frequente, totalizando 53 casos (43\%), seguida por 42 casos de homicídio (34\%) (Gráfico 1). Os indivíduos de cor negra vieram a óbito, principalmente por homicídio (50\%), seguido de acidente e uma menor quantidade por suicídio. As crianças e adolescentes de cor parda tiveram o maior número de óbitos por acidentes (45\%) e homicídio $(37,50 \%)$.
Tabela 2 - Características dos óbitos por causas externas em menores 18 anos, analisados no IML de Cuiabá, entre 2015 e 2016

\begin{tabular}{|c|c|c|}
\hline Variáveis & $\mathbf{N}$ & $\%$ \\
\hline \multicolumn{3}{|l|}{ Causa Morte } \\
\hline Traumatismo Cranioencefálico & 39 & 31,71 \\
\hline Asfixia & 35 & 28,46 \\
\hline Choque hipovolêmico & 19 & 15,45 \\
\hline Indeterminado & 10 & 8,13 \\
\hline Choque séptico & 8 & 6,50 \\
\hline Tamponamento cardíaco & 3 & 2,44 \\
\hline Hemorragia & 2 & 1,63 \\
\hline Sepse & 2 & 1,63 \\
\hline Carbonizado & 1 & 0,81 \\
\hline Desidratado & 1 & 0,81 \\
\hline Insuficiência respiratória & 1 & 0,81 \\
\hline Trombo. Pulmonar & 1 & 0,81 \\
\hline Trauma raquimedular & 1 & 0,81 \\
\hline \multicolumn{3}{|l|}{ Local do óbito } \\
\hline Pública & 82 & 66,67 \\
\hline Domiciliar & 34 & 27,64 \\
\hline Identificação & 7 & 5,69 \\
\hline \multicolumn{3}{|l|}{ Histórico do óbito } \\
\hline Perfuração Arma Fogo & 37 & 30,08 \\
\hline Ac. Trânsito & 27 & 21,95 \\
\hline Afogamento & 16 & 13,01 \\
\hline Broncoaspiração & 8 & 6,50 \\
\hline Queimado & 7 & 5,69 \\
\hline Sem identificação & 6 & 4,88 \\
\hline Envenenamento & 4 & 3,25 \\
\hline Enforcamento & 4 & 3,25 \\
\hline Pneumonia & 3 & 2,44 \\
\hline Choque elétrico & 2 & 1,63 \\
\hline Meningite & 2 & 1,63 \\
\hline Perfuração Arma Branca & 2 & 1,63 \\
\hline Confinamento & 2 & 1,63 \\
\hline Esganadura & 2 & 1,63 \\
\hline Esmagado & 2 & 1,63 \\
\hline Espancamento & 2 & 1,63 \\
\hline Queda & 2 & 1,63 \\
\hline
\end{tabular}


Gráfico 1 - Circunstâncias dos óbitos por causas externas na faixa etária de 0 a 18 anos

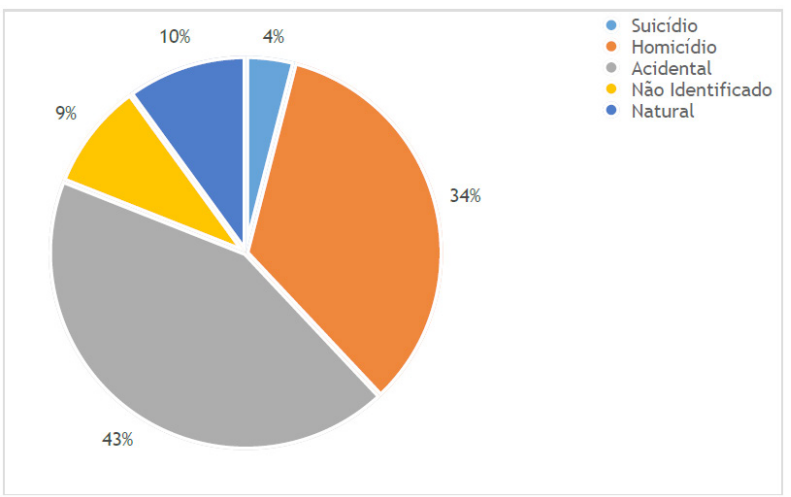

Ao relacionar faixa etária com óbitos causados por traumatismo, foi evidenciado que o risco de morte foi $160 \%$ maior no grupo 13 a 18 anos de idade quando comparado com os de 0 a 12 anos. Além disso, a circunstância do óbito e o local também interferiram no risco de óbito ( $p \leq 0,05)$, sendo que o risco foi $78 \%$ maior por causa acidentais quando comparado com não acidentais, e 5 vezes maior a chance de ocorrer o óbito por traumatismo em via pública. Já o sexo e a cor da pele não interferiram no risco de óbito (Tabela 3).

\section{DISCUSSÃO |}

Mortes por violência e acidentes, há mais de duas décadas, têm sido responsáveis por um número elevado de óbitos em menores de 18 anos, superando até mesmo as mortes por doenças infecciosas ${ }^{7}$.

No presente estudo, os dados demonstraram que essas ainda são as principais causas de óbitos nas crianças e adolescentes, sendo $43 \%$ por circunstâncias acidentais e 34\% por homicídio, o que está em concordância com outros estudos, que referem percentual aproximado de $20 \%$ de mortes por acidentes e de $61,1 \%$ por agressão ${ }^{2,8}$. Em relação à faixa etária, observou-se que os indivíduos mais afetados têm entre 10 e 18 anos, sendo esse predomínio explicado pelo fato de que esses indivíduos são mais vulneráveis à violência em decorrência da criminalidade e da exposição às drogas ${ }^{8,9}$. Já as faixas etárias inferiores, têm uma maior tendência de se envolverem em acidentes, pois as crianças são imaturas, estão em fase de desenvolvimento e costumam ter muitas curiosidades ${ }^{10}$

Ao relacionarmos as desigualdades de sexo com as mortes por causas violentas, foi observado que $66,67 \%$ dos óbitos constatados no periodo foram do sexo masculino, sendo

Tabela 3 - Análise bivariada de óbitos por traumatismo em crianças menores de 18 anos necropsiadas no IML de Cuiabá nos anos de 2015 e 2016

\begin{tabular}{|c|c|c|c|c|c|}
\hline \multirow[b]{2}{*}{ Variáveis explicativas } & \multicolumn{5}{|c|}{ Óbitos causados por traumatismo } \\
\hline & Sim & Não & $\mathbf{R R}$ & $\mathrm{IC} 95 \%$ & $\mathbf{p}$ \\
\hline \multicolumn{6}{|l|}{ Sexo } \\
\hline Masculino & $28(34,15)$ & $54(65,85)$ & 0,85 & $0,4-1,5$ & 0,5876 \\
\hline Feminino & $12(29,27)$ & $29(70,73)$ & 1,0 & & \\
\hline \multicolumn{6}{|l|}{ Faixa etária } \\
\hline $13-18$ anos & $25(52,08)$ & $23(27,71)$ & 2,6 & $1,5-4,4$ & 0,0002 \\
\hline $0-12$ anos & $15(20,0)$ & $60(80,0)$ & 1,0 & & \\
\hline \multicolumn{6}{|l|}{ Cor de pele } \\
\hline Pardos & $28(35,00)$ & $52(65,00)$ & 1,25 & $0,7-2,2$ & 0,42 \\
\hline Não pardos & $12(27,91)$ & $31(72,09)$ & 1,0 & & \\
\hline \multicolumn{6}{|l|}{ Circunstância do óbito } \\
\hline Acidental & $23(43,40)$ & $30(56,60)$ & 1,78 & $1,0-2,9$ & 0,025 \\
\hline Não acidental & $17(24,29)$ & $53(75,71)$ & 1,0 & & \\
\hline \multicolumn{6}{|l|}{ Local do óbito } \\
\hline Via pública & $37(45,12)$ & $45(54,88)$ & 6,16 & $2,0-18,8$ & $<0,01$ \\
\hline Não pública & $3(7,32)$ & $38(92,68)$ & 1,0 & & \\
\hline
\end{tabular}


uma frequência significativamente superior comparado ao feminino. Esse fato está em concordância com outras literaturas, as quais alegam que os homens possuem mais fatores de risco, expondo-se mais a agressões e acidentes de trânsito ${ }^{8,11}$. Normalmente, os homens, mais que as mulheres, consomem bebidas alcoólicas, fazem o uso de drogas ilícitas, estão mais inseridos no mercado de trabalho, seja em atividades lícitas ou não, podendo estar envolvidos com crimes e terem contato com armas de fogo ${ }^{6,12}$.

Os indivíduos de cor parda foram os que apresentaram maior frequência $(65,04 \%)$ de óbitos nesta análise, o que pode ser justificado pela maior quantidade de indivíduos dessa cor no estado do Mato Grosso, de acordo com os dados do IBGE do ano de $2010^{7}$. Com isso, os não brancos são, com mais frequência, vítimas fatais de violência, problemática que se relaciona com as condições socioeconômicas desse grupo ${ }^{1}$.

No entanto, ponto que chamou atenção foi que neste estudo o número de óbitos de indivíduos brancos superou o dos negros. Contrariamente, outros autores relataram que os negros são os mais vulneráveis e costumam ter índices mais altos de óbitos por causas externas ${ }^{1}$.

Embora os menores de 18 anos de cor negra tenham apresentado índice de óbito inferior ao dos demais, observou-se tanto neste estudo como em outros, que os negros continuam morrendo, em sua maioria, por homicídio ${ }^{1,6,13}$. Pesquisas anteriores relacionaram esse fato ao maior envolvimento desses indivíduos com a criminalidade, em vista da desigualdade social e das condições precárias de sobrevivência ${ }^{1}$. A falta de oportunidades de trabalho os levaria a uma ilusão do dinheiro fácil por meio de tráfico de drogas, além da sensação de poder dada pelo uso de arma e o espírito aventureiro próprio dos jovens, sendo esses os fatores que estariam vinculados à inserção dos adolescentes de baixa renda no mundo do crime $e^{6,14}$.

Já os brancos e pardos vieram a óbito, em sua grande maioria, por acidentes e em geral, essa circunstância de morte teve um percentual muito elevado (43\%), levandonos a considerar que não existe uma raça mais vulnerável para tal situação.

Desde a década de 80, os homicídios e acidentes de trânsito eram as principais causas de mortes em crianças e adolescentes ${ }^{15}$. Nesta análise, também foi evidenciado o alto índice de homicídios, causados principalmente por perfuração por arma de fogo, tendo acometido 37 dos 123 indivíduos que vieram a óbito, e crescem devido à disseminação do porte de arma ilegal entre os jovens, o que corrobora para o ambiente mais perigoso e violento ${ }^{1,6}$. Sobre os acidentes de trânsito, observou-se que a tendência de três décadas atrás se manteve, pois continuam sendo responsáveis por uma parcela significativa das mortes nessa faixa etária, com uma frequência de $21,95 \%$, além de um percentual elevado de acidentes com fogo, afogamentos e broncoaspiração ${ }^{6,8,10}$.

As vias onde mais ocorreram os óbitos foram públicas, como consequência do alto percentual de acidentes de trânsito e perfuração por arma de fogo. No entanto, os demais tipos de acidentes, como broncoaspiração e óbito por queimadura, costumam ocorrer dentro do próprio domicílio, o que já foi relatado em outros estudos ${ }^{6}$.

Como resultado do grande percentual de perfuração por arma de fogo e de acidentes de trânsito, o traumatismo foi principal causa de morte encontrada nas crianças e adolescente. Observou-se que o traumatismo cranioencefálico foi o mais frequente $(31,71 \%)$. Sobre o padrão de acometimento, a tendência continua a mesma que a relatada por um estudo realizado no estado do Mato Grosso em $2009^{8}$, e os indivíduos mais vulneráveis estão na faixa etária de 13 a 18 anos e são do sexo masculino.

Ainda analisando os óbitos por traumatismo, em vista da quantidade elevada dos acidentes de trânsito, notou-se que a principal circunstância na qual ocorreram os óbitos foi acidental. Uma das explicações para esse fato se deve à imprudência dos motoristas no trânsito, que, por vezes, estão sob efeito de bebidas alcoólicas e drogas ilícitas, não respeitam leis de trânsito, não fazem uso de cintos de segurança ou até mesmo não possuem habilitação para dirigir ${ }^{8,16}$.

\section{CONCLUSÃO |}

Em vista dos óbitos por causas externas, ressaltado no presente estudo, é de suma importância que isso seja avaliado de forma minuciosa para que medidas possam ser tomadas visando à prevenção desses agravos. Esse aumento não tem sido justificado pela diminuição das mortes por moléstias infecciosas, por exemplo, mas sim porque os jovens estão em contato com a criminalidade cada vez mais 
precocemente, sendo expostos a agressões, perfuração por arma de fogo e acidentes com frequência.

Sabe-se que apesar da evolução socioeconômica obtida nos últimos anos, o Brasil ainda é um país com muita desigualdade social e com áreas de extrema pobreza, e isso propicia a violência e os crimes entre os mais desfavorecidos. Como consequência dessa conjuntura, os homicídios têm sido uma das principais circunstâncias de mortes entre os menores de 18 anos. Os não brancos, do sexo masculino, que estão na faixa etária de 13 a 18 anos, são os que vêm, há anos, sendo mais vulneráveis a essas causas de morte.

Além da violência, as crianças e adolescentes vêm sendo vítimas dos acidentes, especificamente de trânsito, responsáveis por um número significativo de óbitos. Isso reflete uma falha tanto dos cidadãos, que inúmeras vezes são imprudentes no trânsito, como do próprio setor de fiscalização das normas de trânsito, quanto ao uso de cinto de segurança, dirigir sob efeito de álcool ou drogas ilícitas e até mesmo sobre a velocidade permitida nas vias.

Os estudos sobre essa situação vêm contribuindo para o entendimento da problemática, mas não cabe somente isso aos profissionais da saúde. Apesar de não poderem intervir em muitos fatores que levam indivíduos a morrerem por violência e acidentes, esses profissionais são capazes de atuar de forma ativa em movimentos solidários os quais incentivam as crianças e adolescentes a não se envolverem com a criminalidade e as drogas. Os projetos sociais voltados para essas faixas etárias têm obtido êxito, mas necessitam estar presentes em uma grande área de todo o País.

\section{REFERÊNCIAS |}

1. Minayo MCS. A violência na adolescência: um problema de saúde pública. Cad Saúde Pública. 1990; 6(3):278-92.

2. DATASUS [Internet]. Informações de saúde: sistema de informações sobre mortalidade [acesso em 10 jun 2018]. Disponível em: http://www.datasus.gov.br.

3. Minayo MCS, Souza ER. Violência para todos. Cad Saúde Pública. 1993; 9(1):65-78.
4. Brasil. Lei no ${ }^{\circ}$ 8.069, de 13 de julho de 1990. Dispõe sobre o Estatuto da Criança e do Adolescente e dá outras providências. Diário Oficial da União 16 jul 1990.

5. Brasil. Ministério da Saúde. Violência faz mal à saúde. Brasília: Ministério da Saúde; 2006.

6. Barros MDA, Ximenes RL, Maria LC. Mortalidade por causas externas em crianças e adolescentes: tendências de 1979 a 1995. Rev Saúde Pública. 2001; 35(2):142-9.

7. Instituto Brasileiro de Geografia e Estatística. Censo demográfico: características gerais da população, religião e pessoas com deficiência. Rio de Janeiro: IBGE; 2012.

8. Matos KF, Martins CBG. Perfil epidemiológico da mortalidade por causas externas em crianças, adolescentes e jovens na capital do Estado de Mato Grosso, Brasil, 2009. Epidemiol Serv Saúde. 2012; 21(1):43-53.

9. Fonzar UJV. Análise espacial da mortalidade por causas externas do município de Maringá. Acta Sci Health Sci. 2008; 30(2):145-54.

10. Martins CBG, Andrade SM. Causas externas entre menores de 15 anos em cidade do Sul do Brasil: atendimentos em pronto-socorro, internações e óbitos. Rev Bras Epidemiol. 2005; 8(2):194-204.

11. Costa IER, Ludermir AB, Avelar I. Violência contra adolescentes: diferenciais segundo estratos de condição de vida e sexo. Ciênc Saúde Coletiva. 2007; 12(5):1193-200.

12. Valença Neto PF, Siqueira BPJ, Nery AA, Casotti CA. Tendência da mortalidade masculina por causas externas. Rev Enferm UFPE. 2015; 9(5):7877-86.

13. Agranonik M, Furstenau CR, Bandeira MD. Aspectos da mortalidade de crianças e adolescentes por causas externas no RS, em 2000-14. Indic Econ FEE. 2017; 44(4):53-64

14. Minayo MCS. Violência social sob a perspectiva da saúde pública. Cad Saúde Pública. 1994; 10(Supl. 1):S7-S18.

15. Assis SG. Crianças e adolescentes violentados: passado, presente e perspectivas para o futuro. Cad Saúde Pública. 1994; 10:S126-S34. 
16. Santos WN, Silva RAR, Figueiredo TAM, Coqueiro JM. Fatores de riscos e estratégias preventivas para os acidentes de trânsito: revisão integrativa. Rev Enferm UFPE. 2016; 10(9):3463-72.

Correspondencia para/Reprint request to:

Igor Lucas Pinheiro de Sousa

Av. Desembargador Antônio Querino de Araújo, 1374,

Poção, Cuiabá/MT, Brasil

CEP: 78015-580

E-mail:igor_lucas9@botmail.com

Recebido em: 02/04/2019

Aceito em: 04/09/2020 